\title{
Numerical determination of OPE coefficients in the 3D Ising model from off-critical correlators.
}

\author{
M. Caselle ${ }^{1}$, G. Costagliola ${ }^{1}$, N. Magnoli ${ }^{2}$ \\ ${ }^{1}$ Dipartimento di Fisica, Università di Torino and INFN, Via P. Giuria 1, 10125, Torino, Italy. \\ ${ }^{2}$ Dipartimento di Fisica, Università di Genova and INFN, Via Dodecaneso 33, 16146, Genova, Italy.
}

(Dated: July 17, 2018)

\begin{abstract}
We propose a general method for the numerical evaluation of OPE coefficients in three dimensional Conformal Field Theories based on the study of the conformal perturbation of two point functions in the vicinity of the critical point. We test our proposal in the three dimensional Ising Model, looking at the magnetic perturbation of the $\langle\sigma(\mathbf{r}) \sigma(0)\rangle,\langle\sigma(\mathbf{r}) \epsilon(0)>$ and $\langle\epsilon(\mathbf{r}) \epsilon(0)>$ correlators from which we extract the values of $C_{\sigma \epsilon}^{\sigma}=1.07(3)$ and $C_{\epsilon \epsilon}^{\epsilon}=1.45(30)$. Our estimate for $C_{\sigma \epsilon}^{\sigma}$ agrees with those recently obtained using conformal bootstrap methods, while $C_{\epsilon \epsilon}^{\epsilon}$, as far as we know, is new and could be used to further constrain conformal bootstrap analyses of the $3 \mathrm{~d}$ Ising universality class.
\end{abstract}

\section{INTRODUCTION}

In the past few years a renewed interest in the Conformal Bootstrap approach [1] to three dimensional Conformal Field Theories (CFT) led to a set of remarkable results [2]-[19] on the universality classes of several 3d statistical models. Among these models a particular attention has been devoted to the $3 \mathrm{~d}$ Ising case, both for its physical relevance and for the fact that in this case very precise numerical estimates exist for the scaling dimensions [20], which allowed highly non trivial tests of the conformal bootstrap results. It would be interesting to perform a similar comparison also for the Operator Product Expansion (OPE) coefficients [21], but for these constants Monte Carlo estimates with an accuracy comparable with that of the scaling dimensions are still lacking. As an attempt to fill this gap we propose in this paper a general strategy for the numerical estimate of the structure constants and, as a proof of concept, we evaluate $C_{\sigma \epsilon}^{\sigma}$ and $C_{\epsilon \epsilon}^{\epsilon}$ in the Ising case. Our estimates should be considered mainly as an exploratory study on the effectiveness of the procedure with a limited use of computer power. We plan in a forthcoming paper to obtain more precise estimates for these and other OPE coefficients.

It is well known that estimating structure constants is much more difficult than estimating scaling dimensions. The most naive approach would be to extract them directly from three point functions (i.e. the connected correlators of three operators) at criticality. However it is easy to see that the subtraction in the connected correlator of the two point components leads to a mix of contributions with similar exponents which makes it very difficult to extract the sought for OPE coefficients. In this letter we propose an alternative method based on conformal perturbation which allows to overcome this problem. The main idea is to study the perturbed two point functions using the OPE to rewrite them as sums of perturbed one point functions and then use the known scaling behaviour of one point functions and structure constants to write the perturbed two point function as an expansion in powers of the scaling variable. Following [21] it is always possible to write the short distance behaviour of two-point correlators in the vicinity of a critical point as a short distance expansion:

$$
<O_{i}(\mathbf{r}) O_{j}(\mathbf{0})>_{h}=\sum_{k} C_{i j}^{k}(r ; h)<O_{k}(\mathbf{0})>_{h}
$$

where $<. .>_{h}$ denotes the expectation values with respect to the perturbed action, the $O_{i}$ represent a complete set of operators of the conformal theory and the $C_{i j}^{k}(r, h)$ are the Wilson coefficients. While the one point functions $<O_{k}(\mathbf{0})>_{h}$ cannot be determined from the knowledge of the critical correlators and take care of the non-perturbative physics of the model, the Wilson coefficients can be obtained from integrals of critical correlators. Moreover it was shown in [22] that the $n^{\text {th }}$ order derivatives of the Wilson coefficients with respect to the perturbing parameter of the theory are always infrared finite. The structure constants can then be easily extracted from the coefficients of the short distance expansion (see [22-24] for further details). In practice one is limited for numerical reasons to the first few coefficients (typically the first two or three) which however (combining all the possible two points functions and relevant perturbations) are enough to obtain all the structure constants among relevant operators.

As it is easy to see the method is based on very general features of CFTs and OPEs. It can be applied to any d-dimensional CFT and in fact it was applied a few years ago to the $2 \mathrm{~d}$ Ising model perturbed by a magnetic field [23, 24], to various perturbations of the Tricritical Ising model [25] and then used to identify the signatures of subleading irrelevant operators in the perturbed two point functions [26]. In all these cases the structure constants were already known from the exact solution of Belavin Polyakov and Zamolodchikov [27] and the main emphasis was on comparing the perturbative results with the numerical simulations.

The aim of this paper is to apply this strategy to the three dimensional case. In particular we shall address, as an example, the magnetic perturbation of the $3 \mathrm{~d}$ Ising model. 


\section{ISING MODEL PERTURBED BY A MAGNETIC FIELD $h$}

The action of the continuum Ising model in $3 D$ perturbed by a magnetic field is:

$$
S=S_{c f t}+h \int \sigma(\mathbf{r}) d \mathbf{r} .
$$

where $S_{c f t}$ is the action of the conformal field theory which describes the model at the critical point.

At the conformal point there are two quasi-primary relevant fields $\sigma$ and $\epsilon$, whose dimensions are given by $\Delta_{\sigma}=$ 0.51814(5), $\Delta_{\epsilon}=1.41275(25)$ [20].

The VEVs acquire a dependence on $h$, that can be fixed using renormalization group arguments:

$$
\begin{aligned}
& <\sigma>=A_{\sigma} h^{\frac{\Delta_{\sigma}}{\Delta_{h}}} \\
& <\epsilon>=A_{\epsilon} h^{\frac{\Delta_{\epsilon}}{\Delta_{h}}}
\end{aligned}
$$

In order to simplify notations let us introduce the scaling variable $t=|h| r^{\Delta_{h}}$, and an adimensional version of the structure constants and their derivatives:

$$
\begin{aligned}
C_{i j}^{k} & \equiv \lim _{r->\infty} C_{i j}^{k}(r ; 0) r^{\operatorname{dim}\left(C_{i j}^{k}(r ; 0)\right)} \\
\partial_{h} C_{i j}^{k} & \equiv \lim _{r->\infty} \partial_{h} C_{i j}^{k}(r ; 0) r^{\operatorname{dim}\left(\partial_{h} C_{i j}^{k}(r ; 0)\right)}
\end{aligned}
$$

With these definitions the short distance expansion of $\sigma$ and $\epsilon$ correlators becomes:

$$
\begin{aligned}
r^{2 \Delta_{\sigma}}<\sigma(\mathbf{r}) \sigma(0)> & =C_{\sigma \sigma}^{1}+A_{\epsilon} C_{\sigma \sigma}^{\epsilon} t^{\frac{\Delta_{\epsilon}}{\Delta_{h}}} \\
& +A_{\sigma} \partial_{h} C_{\sigma \sigma}^{\sigma} t^{\frac{\Delta_{\sigma}}{\Delta_{h}}+1}+O\left(t^{2}\right) \\
r^{\Delta_{\sigma}+\Delta_{\epsilon}}<\sigma(\mathbf{r}) \epsilon(0)> & =A_{\sigma} C_{\sigma \epsilon}^{\sigma} t^{\frac{\Delta_{\sigma}}{\Delta_{h}}}+\partial_{h} C_{\sigma \epsilon}^{1} t \\
& +A_{\epsilon} \partial_{h} C_{\sigma \epsilon}^{\epsilon} t^{\frac{\Delta_{\epsilon}}{\Delta_{h}}+1}+O\left(t^{\frac{\Delta_{\sigma}}{\Delta_{h}}+2}\right) \\
r^{2 \Delta_{\epsilon}}<\epsilon(\mathbf{r}) \epsilon(0)> & =C_{\epsilon \epsilon}^{1}+A_{\epsilon} C_{\epsilon \epsilon}^{\epsilon} t^{\frac{\Delta_{\epsilon}}{\Delta_{h}}} \\
& +A_{\sigma} \partial_{h} C_{\epsilon \epsilon}^{\sigma} t^{\frac{\Delta_{\sigma}}{\Delta_{h}}+1}+O\left(t^{2}\right)
\end{aligned}
$$

where $\Delta_{h}=3-\Delta_{\sigma}=2.48186(5)$ is the dimension of the magnetic field and with the standard CFT normalizations we may set $C_{\epsilon \epsilon}^{1}=C_{\sigma \sigma}^{1}=1$.

\section{Conversion from lattice to continuum normalizations.}

The partition function of the $3 \mathrm{~d}$ Ising model is given by:

$$
Z=\sum_{\sigma_{i}= \pm 1} e^{\beta\left(\sum_{<i, j>} \sigma_{i} \sigma_{j}+H \sum_{i} \sigma_{i}\right)}
$$

where $\langle i, j\rangle$ indicates nearest neighbors sites in the lattice which we assume to be a three-dimensional cubic lattice of size $L$. Fixing $\beta$ at its critical value $\beta_{c}=$ 0.2216544 [28] and defining $h_{l}=\beta_{c} H$, we have:

$$
Z=\sum_{\sigma_{i}= \pm 1} e^{\beta_{c} \sum_{<i, j>} \sigma_{i} \sigma_{j}+h_{l} \sum_{i} \sigma_{i}}
$$

It is natural to define the lattice discretization of $\sigma$ as $\sigma^{l} \equiv \frac{1}{L^{3}} \sum_{i} \sigma_{i}$, so that its mean value coincides with the magnetization. In a similar way one can define the energy operator $\epsilon^{l}$ as $\epsilon^{l} \equiv \frac{1}{3 L^{3}} \sum_{<i, j>} \sigma_{i} \sigma_{j}-\epsilon_{c r}$, where $\epsilon_{c r}$ is the energy at the critical point, so that the mean value of $\epsilon^{l}$ coincides with the singular part of the internal energy.

It is important to notice that measuring the mean values (3/4) and the two point functions 55/6,7) on the lattice we find the lattice versions of the amplitudes $A$ and structure constants $C$ (which we shall denote in the following with the index $l: A^{l}$ and $C^{l}$ ). To relate these constants with the continuum ones we must first fix the relative normalization of $\sigma^{l}, \epsilon^{l}$ and $h_{l}$ with respect to $\sigma, \epsilon$ and $h$. The simplest way to do this is by comparing the correlators at the critical point. In fact from

$$
<\sigma_{i} \sigma_{j}>=\frac{R_{\sigma}^{2}}{\left|r_{i j}\right|^{2 \Delta_{\sigma}}},
$$

we get (assuming the standard normalization of the two point function in the continuum) $\sigma^{l}=R_{\sigma} \sigma$. By considering the $\epsilon^{l}$ correlator we get similarly $\epsilon^{l}=R_{\epsilon} \epsilon$. Finally, $h_{l}$ is fixed by matching the lattice and continuum perturbation terms: $h_{l}=R_{\sigma}^{-1} h$.

Using $R_{\sigma}$ and $R_{\epsilon}$ we can easily obtain continuum amplitudes and OPE constants from the lattice ones. For example from the equation (4) we get $A_{\epsilon}^{l}=R_{\sigma}{ }^{\frac{\Delta \epsilon}{\Delta h}} R_{\epsilon} A_{\epsilon}$ and from this and the equations (5) and (10) we have the rescaling for the structure constant: $C_{\sigma \sigma}^{\epsilon}{ }^{l} R_{\epsilon}=C_{\sigma \sigma}^{\epsilon}$.

\section{MONTE CARLO SIMULATIONS}

\section{Estimating $R_{\sigma}$ and $R_{\epsilon}$}

As a first step we extracted the values of $R_{\sigma}$ and $R_{\epsilon}$ from a finite size scaling analysis of the two point correlators at the critical point. The table below summarizes our results. We are not aware of independent estimates of these quantities except for $\epsilon_{c r}$ which was estimated in [29] as $\epsilon_{c r}=0.3302022(5)$ in good agreement with our result. The quoted uncertainties combine both statistical and systematic errors. It should be noticed, in view of possible future improvements of our analysis, that the main source of error was due to the infinite volume extrapolation of our results (we used lattices in the range $120 \leq L \leq 200$ ). 
Table I. Rescaling and other useful constants

\begin{tabular}{ll}
\hline$R_{\sigma}$ & $0.550(4)$ \\
$R_{\epsilon}$ & $0.237(3)$ \\
$A_{\sigma}^{l}$ & $1.0125(5)$ \\
$A_{\epsilon}^{l}$ & $0.608(1)$ \\
$\epsilon_{c r}$ & $0.330213(12)$ \\
\hline
\end{tabular}

\section{Perturbed correlators}

To extract the structure constants we then estimated the same correlators in presence of a small magnetic field $h_{l}$. The choice of $h_{l}$ is constrained by two main requirements: $h_{l}$ should be small enough to keep the correlation length $\xi$ as large as possible, at the same time it should not be too small to avoid finite size effects. The optimal choice is thus fixed by the maximal lattice size which we could simulate with the computer resources at our disposal, which was fixed to be $L=200$. Given this constraint the optimal range turned out to be $2 \cdot 10^{-5}<h_{l}<0.75 \cdot 10^{-5}$. In this range the correlation length spans from about 20 to 40 lattice spacings, allowing to sample a sufficient number of different distances of the correlators.

For all values of $h$ we performed our simulations on lattices of size $L=200$ using a state of art Monte Carlo algorithm with about $10^{7}$ configurations for each simulation. We fitted the correlators with eq.s (5, 6, 7), setting $C_{\sigma \sigma}^{1}=C_{\epsilon \epsilon}^{1}=1$, using $A_{\sigma}, A_{\epsilon}, \Delta_{\sigma}, \Delta_{\epsilon}$ as fixed inputs and keeping as only free parameters the structure constants. It turned out that in all correlators the last terms quoted in eq.s (5, 7) and the last two terms in eq. 6 were negligible within the errors and in all three cases we ended up with a linear fit with only one free parameter. A major source of uncertainty in our estimates is the systematic error due to the uncertainty in the estimates of $R_{\sigma}$ and $R_{\epsilon}$. We quote separately in the following these errors (which we report in square brackets) from the statistical ones. Due to the different scaling behaviour, the relative size of statistical and systematic errors is different for the three correlators. For $C_{\sigma \sigma}^{\epsilon}$ estimated from $<\sigma(\mathbf{r}) \sigma(0)>$ the two errors are of similar magnitude. $C_{\epsilon \epsilon}^{\epsilon}$ extracted from $<\epsilon(\mathbf{r}) \epsilon(0)>$ is dominated by the systematic error while for $C_{\sigma \epsilon}^{\sigma}$. extracted from $<\sigma(\mathbf{r}) \epsilon(0)>$ the systematic error is negligible (due to the fact that the critical correlator vanishes in this case).

The tables below report our results for the structure constants. $r_{\min }$ and $r_{\max }$ denote the range of distances included in the fit. In all cases we chose $r_{\min }=5$. We verified that in all three cases this was enough to eliminate lattice artifacts and to neglect short distance subleading contributions in the correlators.

Looking at tab.s (IIIV) we see that, within our statistical errors $C_{\sigma \sigma}^{\epsilon}=C_{\sigma \epsilon}^{\sigma}$. Combining the results listed in tab.s (IIIIIIV] we quote as our final estimate for the two structure constants $C_{\sigma \epsilon}^{\sigma}=1.07(3)$ and $C_{\epsilon \epsilon}^{\epsilon}=1.45(30)$. The first value is in good agreement with a recent conformal
Table II. Results for the structure constant $C_{\sigma \sigma}^{\epsilon}$ obtained from the spin-spin correlator. We report systematic errors in square brackets.

\begin{tabular}{llll}
\hline$h_{l}$ & $r_{\min }$ & $r_{\max }$ & $C_{\sigma \sigma}^{\epsilon}$ \\
\hline $2 \cdot 10^{-5}$ & 6 & 20 & $1.06(3)[7]$ \\
$10^{-5}$ & 5 & 35 & $1.04(2)[7]$ \\
$0.85 \cdot 10^{-5}$ & 5 & 35 & $1.06(3)[8]$ \\
$0.75 \cdot 10^{-5}$ & 5 & 40 & $1.07(3)[8]$ \\
\hline
\end{tabular}

Table III. Results for the structure constant $C_{\epsilon \epsilon}^{\epsilon}$ obtained from the energy-energy correlator. We report systematic errors in square brackets.

\begin{tabular}{llll}
\hline$h_{l}$ & $r_{\min }$ & $r_{\max }$ & $C_{\epsilon \epsilon}^{\epsilon}$ \\
\hline $2 \cdot 10^{-5}$ & 5 & 16 & $1.47(10)[30]$ \\
$10^{-5}$ & 5 & 18 & $1.42(10)[50]$ \\
$0.85 \cdot 10^{-5}$ & 5 & 18 & $1.43(12)[50]$ \\
\hline
\end{tabular}

bootstrap calculation [19]: $\left(C_{\sigma \epsilon}^{\sigma}\right)^{2}=1.10636(9)$ [30]. We are not aware of any estimate for the second constant $C_{\epsilon \epsilon}^{\epsilon}$. The very fact that it is different from zero is rather non trivial. In fact in the two dimensional model $C_{\epsilon \epsilon}^{\epsilon}=0$ as a consequence of dual symmetry. Our calculation shows that indeed this is not a generic property of the Ising model but is a specific feature of the two dimensional self-dual case.

\section{CONCLUDING REMARKS}

In this paper we proposed a general strategy for the Monte Carlo estimate of OPE coefficients in d-dimensional spin models. As a proof of concept of our method we performed an exploratory study in the $3 \mathrm{~d}$ Ising case and found a value of $C_{\sigma \epsilon}^{\sigma}$ in agreement with the known conformal bootstrap results and a preliminary estimate for $C_{\epsilon \epsilon}^{\epsilon}$ which could be used as input for further constrain existing conformal bootstrap calculations. Similarly, the knowledge of these structure constants could be used to constrain the linear response dynamics at finite temperature of Conformal Quantum Critical systems [32].

We see a few possible improvements of our approach:

- The systematic uncertainty which is the major source of error in $C_{\epsilon \epsilon}^{\epsilon}$ could be improved, following the approach proposed in [20], looking at different realizations of the $3 \mathrm{~d}$ universality class with improved scaling behaviour.

- It would be useful to combine the magnetic perturbation that we studied in this paper with other types of perturbation.

- It would be interesting to extend the method also to boundary CFTs [8] and to CFTs containing conformal defects [33, 34]

We plan to address these issues in a forthcoming publication. 
Table IV. Results for the structure constant $C_{\sigma \epsilon}^{\sigma}$ obtained from the spin-energy correlator.

\begin{tabular}{llll}
\hline$h_{l}$ & $r_{\min }$ & $r_{\max }$ & $C_{\sigma \epsilon}^{\sigma}$ \\
\hline $2 \cdot 10^{-5}$ & 5 & 16 & $1.10(3)$ \\
$10^{-5}$ & 5 & 16 & $1.07(4)$ \\
$0.85 \cdot 10^{-5}$ & 5 & 14 & $1.07(5)$ \\
\hline
\end{tabular}

Acknowledgements We thank F. Gliozzi, M. Hasenbusch, A. Rago, S. Rychkov and W. Witczak-Krempa for useful discussions and suggestions.

[1] A. M. Polyakov, Zh. Eksp. Teor. Fiz. 66 (1974) 23-42.

[2] R. Rattazzi, V. S. Rychkov, E. Tonni and A. Vichi, JHEP 0812 (2008) 031 [arXiv:0807.0004 [hep-th]].

[3] V. S. Rychkov and A. Vichi, Phys. Rev. D 80 (2009) 045006 [arXiv:0905.2211 [hep-th]].

[4] R. Rattazzi, S. Rychkov and A. Vichi, Phys. Rev. D 83 (2011) 046011 arXiv:1009.2725 [hep-th]].

[5] D. Poland and D. Simmons-Duffin, JHEP 1105 (2011) 017 arXiv:1009.2087 [hep-th]].

[6] S. El-Showk, M. F. Paulos, D. Poland, S. Rychkov, D. Simmons-Duffin and A. Vichi, Phys. Rev. D 86 (2012) 025022 [arXiv:1203.6064 [hep-th]].

[7] D. Pappadopulo, S. Rychkov, J. Espin and R. Rattazzi, Phys. Rev. D 86 (2012) 105043 [arXiv:1208.6449 [hep-th]].

[8] P. Liendo, L. Rastelli and B. C. van Rees, JHEP 1307 (2013) 113 [arXiv:1210.4258 [hep-th]].

[9] S. El-Showk and M. F. Paulos, Phys. Rev. Lett. 111 (2013) 24, 241601 [arXiv:1211.2810 [hep-th]].

[10] Z. Komargodski and A. Zhiboedov, JHEP 1311 (2013) 140 [arXiv:1212.4103 [hep-th]].

[11] R. Rattazzi, S. Rychkov and A. Vichi, J. Phys. A 44 (2011) 035402 [arXiv:1009.5985 [hep-th]].

[12] D. Poland, D. Simmons-Duffin and A. Vichi, JHEP 1205 (2012) 110 [arXiv:1109.5176 [hep-th]].

[13] A. Vichi, JHEP 1201 (2012) 162 [arXiv:1106.4037 [hepth]].

[14] F. Gliozzi, Phys. Rev. Lett. 111 (2013) 161602 [arXiv:1307.3111].

[15] F. Gliozzi and A. Rago, JHEP 1410 (2014) 42 [arXiv:1403.6003 [hep-th]].
[16] F. Kos, D. Poland and D. Simmons-Duffin, JHEP 1406 (2014) 091 [arXiv:1307.6856 [hep-th]].

[17] S. El-Showk, M. Paulos, D. Poland, S. Rychkov, D. Simmons-Duffin and A. Vichi, Phys. Rev. Lett. 112 (2014) 141601 [arXiv:1309.5089 [hep-th]].

[18] F. Kos, D. Poland and D. Simmons-Duffin, JHEP 1411 (2014) 109 [arXiv:1406.4858 [hep-th]].

[19] S. El-Showk, M. F. Paulos, D. Poland, S. Rychkov, D. Simmons-Duffin and A. Vichi, J. Stat. Phys. 157 (2014) 869 [arXiv:1403.4545 [hep-th]].

[20] M. Hasenbusch, Phys. Rev. B 82 (2010) 174433. [arXiv:1004.4486

[21] K. G. Wilson, Phys. Rev. 179, 1499 (1969).

[22] R. Guida and N. Magnoli, Nucl. Phys. B 471, 361 (1996) [hep-th/9511209].

[23] M. Caselle, P. Grinza and N. Magnoli, Nucl. Phys. B 579 (2000) 635 [hep-th/9909065].

[24] R. Guida and N. Magnoli, Nucl. Phys. B 483, 563 (1997) [hep-th/9606072].

[25] R. Guida and N. Magnoli, Int. J. Mod. Phys. A 13, 1145 (1998) [hep-th/9612154].

[26] M. Caselle, P. Grinza and N. Magnoli, J. Phys. A 34 (2001) 8733 [hep-th/0103263].

[27] A. M. Polyakov, A. A. Belavin and A. B. Zamolodchikov, J. Statist. Phys. 34, 763 (1984).

[28] A. M. Talapov, H. W. J. Blöte, cond-mat/9603013, J. Phys. A 29 (1996) 5727

[29] M. Hasenbusch, Phys. Rev. B 85 (2012) 174421. [arXiv:1202.6206]

[30] As far as we know the only other existing estimate for this constant was obtained by Henkel [31] in the Hamiltonian limit. A puzzling feature of his result is that, in disagreement with our finding and with [19] he found different values for $C_{\sigma \sigma}^{\epsilon}$ and $C_{\sigma \epsilon}^{\sigma}$. While his value for $C_{\sigma \epsilon}^{\sigma}$ agrees within the errors with our result, $C_{\sigma \sigma}^{\epsilon}$ is incompatible. As noticed in [19], this is most probably due to the fact that the Hamiltonian setting of Henkel's calculation is not Weyl equivalent to the flat $3 \mathrm{~d}$ space in which both our simulations and the bootstrap calculations are performed..

[31] M. Henkel, J.Phys. A20 (1987) 3969.

[32] William Witczak-Krempa, arXiv:1501.03495 [condmat.str-el].

[33] M. Billo', M. Caselle, D. Gaiotto, F. Gliozzi, M. Meineri and R. Pellegrini, JHEP 1307 (2013) 055 [arXiv:1304.4110 [hep-th]].

[34] D. Gaiotto, D. Mazac and M. F. Paulos, JHEP 1403 (2014) 100 [arXiv:1310.5078[hep-th]]. 